\title{
The short-duration GRB 050724 host galaxy in the context of the long-duration GRB hosts ${ }^{\star}$
}

\author{
J. Gorosabel ${ }^{1}$, A. J. Castro-Tirado ${ }^{1}$, S. Guziy ${ }^{1}$, A. de Ugarte Postigo ${ }^{1}$, D. Reverte ${ }^{1}$, A. Antonelli ${ }^{2}$, \\ S. Covino ${ }^{3}$, D. Malesani ${ }^{4}$, D. Martín-Gordón ${ }^{1}$, A. Melandri' ${ }^{2}$, M. Jelínek ${ }^{1}$, \\ N. Elias de la Rosa ${ }^{5}$, O. Bogdanov ${ }^{6}$, and J. M. Castro Cerón ${ }^{7}$
}

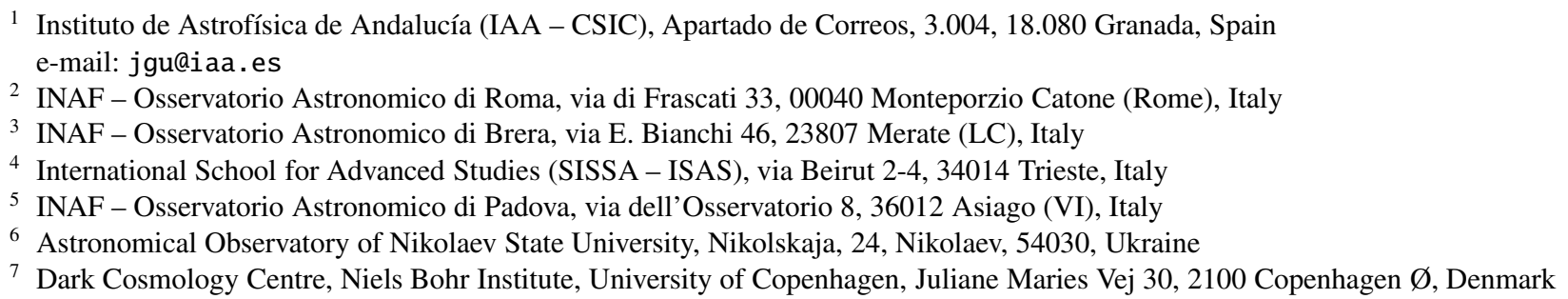

Received 18 October 2005 / Accepted 13 December 2005

\section{ABSTRACT}

We report optical and near-infrared broad band observations of the short-duration GRB 050724 host galaxy, used to construct its spectral energy distribution (SED). Unlike the hosts of long-duration gamma-ray bursts (GRBs), which show younger stellar populations, the SED of the GRB 050724 host galaxy is optimally fitted with a synthetic elliptical galaxy template based on an evolved stellar population (age 2.6 Gyr). The SED of the host is difficult to reproduce with non-evolving metallicity templates. In contrast, if the short GRB host galaxy metallicity enrichment is considered, the synthetic templates fit the observed SED satisfactorily. The internal host extinction is low ( $\left.A_{\mathrm{v}} \lesssim 0.4 \mathrm{mag}\right)$ so it cannot explain the faintness of the afterglow. This short GRB host galaxy is more massive $\left(\sim 5 \times 10^{10} M_{\odot}\right)$ and luminous $\left(\sim 1.1 L^{\star}\right)$ than most of the long-duration GRB hosts. A statistical comparison based on the ages of short- and long-duration GRB host galaxies strongly suggests that short-duration GRB hosts contain, on average, older progenitors. These findings support a different origin for short- and long-duration GRBs.

Key words. gamma rays: bursts - Galaxy: fundamental parameters - techniques: photometric

\section{Introduction}

It is widely accepted nowadays that long-duration gamma-ray bursts (GRBs) are related to core-collapse supernovae (Stanek et al. 2003; Hjorth et al. 2003); the origin of short-duration GRBs is, however, still a mystery (see Kouveliotou et al. 1993, for the definition of short GRBs). Up to 2005 efforts to detect short-duration GRBs at longer wavelengths remained unfruitful (see Hurley et al. 2002; Gorosabel et al. 2002, and references therein), but with prompt and accurate localizations recently reported for four short-duration GRBs by the Swift and HETE-2 satellites (GRB 050509B, Gehrels et al. 2005; GRB 050709, Butler et al. 2005; GRB 050724, Covino et al. 2005; and

* Based on observations made with the Telescopio Nazionale Galileo (Prop. ID TAC-1238) and the Nordic Optical Telescope, operated on the island of La Palma, in the Spanish Observatorio del Roque de los Muchachos of the Instituto de Astrofísica de Canarias, and on observations carried out at the Centro Astronómico Hispano Alemán (CAHA) at Calar Alto operated jointly by the Max-Planck Institut für Astronomie and the Instituto de Astrofísica de Andalucía (CSIC).
GRB 050813, Retter et al. 2005), the field is currently undergoing a rapid breakthrough.

Interestingly enough, for the above mentioned four shortduration GRBs, relatively bright galaxies $(18 \lesssim R \lesssim 24)$ have been found close, or within, the corresponding X-ray error boxes (Bloom et al. 2006; Covino et al. 2006; Hjorth et al. 2005; Antonelli et al. 2005; Gladders et al. 2005; see also Prochaska et al. 2005a). Recent statistical studies (Tanvir et al. 2005; Gal-Yam et al. 2005a) suggesting an excess of bright galaxies coincident with short GRB error boxes support this correlation.

The short GRB 050724 was detected by Swift on 24 July 2005, at 12:34:09 UT. It showed an X-ray afterglow consistent with the location of a bright galaxy present on the Digitized Sky Survey (Antonelli et al. 2005; Bloom et al. 2005), at a redshift of $z=0.257$ (Prochaska et al. 2005b,c). Optical (Gal-Yam et al. 2005b), near-infrared (NIR; Cobb \& Bailyn 2005), and radio (Berger et al. 2005) transients were detected offset $\sim 3 \mathrm{kpc}$ from the host, a value similar to that measured for the GRB 050709 afterglow (Hjorth et al. 2005). 
Table 1. Optical and NIR photometric observations of the host of GRB 050724. The magnitudes are not corrected for Galactic extinction.

\begin{tabular}{|c|c|c|c|c|c|c|c|c|}
\hline $\begin{array}{l}\text { Telescope } \\
\text { (+Instrument) }\end{array}$ & Filter & $\begin{array}{c}\text { Date UT } \\
\text { (mid exposure) }\end{array}$ & $\begin{array}{l}T_{\exp } \\
(\mathrm{s})\end{array}$ & $\begin{array}{l}\text { Seeing } \\
\left({ }^{\prime \prime}\right)\end{array}$ & $\begin{array}{c}\text { Effective } \\
\text { wavelength }(\AA)\end{array}$ & $\begin{array}{l}\text { Bandpass } \\
\text { width }(\AA)\end{array}$ & $\begin{array}{c}\text { Magnitude } \\
\text { (Vega) }\end{array}$ & $\begin{array}{c}\text { Magnitude } \\
\text { (AB) }\end{array}$ \\
\hline $2.5 \mathrm{NOT}$ (+ALFOSC) & $U$ & $6.903 / 08 / 05$ & 900 & 1.3 & 3660 & 355 & $>22.20^{\dagger}$ & $>23.09^{\dagger}$ \\
\hline $2.5 \mathrm{NOT}(+\mathrm{ALFOSC})$ & $B$ & $6.884 / 08 / 05$ & 600 & 1.3 & 4384 & 700 & $22.47 \pm 0.12$ & $22.34 \pm 0.12$ \\
\hline 2.5NOT (+ALFOSC) & $V$ & $6.921 / 08 / 05$ & 600 & 1.2 & 5368 & 527 & $20.69 \pm 0.05$ & $20.69 \pm 0.05$ \\
\hline 2.5NOT (+ALFOSC) & $R$ & $6.929 / 08 / 05$ & 600 & 1.5 & 6627 & 768 & $19.58 \pm 0.03$ & $19.83 \pm 0.03$ \\
\hline 3.6TNG (+NICS) & $J_{\mathrm{s}}$ & $4.872 / 08 / 05$ & $20 \times 60$ & 1.1 & 12541 & 964 & $16.88 \pm 0.04^{\star}$ & $17.83 \pm 0.04^{\star}$ \\
\hline 3.6TNG (+NICS) & $J_{\mathrm{s}}$ & $12.887 / 08 / 05$ & $25 \times 60$ & 1.0 & 12541 & 964 & & \\
\hline 3.5CAHA (+OMEGA 2000$)$ & $J$ & $25.868 / 07 / 05$ & $30 \times 90$ & 2.5 & 12861 & 1713 & $16.87 \pm 0.13^{\ddagger}$ & $17.84 \pm 0.13^{\ddagger}$ \\
\hline 3.6TNG $(+\mathrm{NICS})$ & $H$ & $4.911 / 08 / 05$ & $21 \times 60$ & 1.0 & 16289 & 173 & $15.86 \pm 0.05$ & $17.24 \pm 0.05$ \\
\hline 3.6TNG (+NICS) & $K_{\mathrm{s}}$ & $4.892 / 08 / 05$ & $21 \times 60$ & 0.9 & 21203 & 204 & $15.03 \pm 0.04^{\star}$ & $16.86 \pm 0.04^{\star}$ \\
\hline 3.6TNG (+NICS) & $K_{\mathrm{s}}$ & $12.863 / 08 / 05$ & $25 \times 60$ & 1.1 & 21203 & 204 & & \\
\hline
\end{tabular}

$\ddagger$ Only used for checking the afterglow contamination; $\dagger 3 \sigma$ upper limit; $\star$ combined images from two epochs.

The study of the spectral energy distribution (SED) of GRB host galaxies provides information on the star-formation rate (SFR), the stellar mass content, the stellar population age, the absolute luminosity, and the extinction. In the present paper we construct the SED of the GRB 050724 host galaxy and compare its properties with the ones reported for the hosts of the longduration GRBs (Chary et al. 2002; Christensen et al. 2004; Gorosabel et al. 2005a, and references therein). Throughout this paper we assume a cosmology where $\Omega_{\Lambda}=0.7, \Omega_{\mathrm{M}}=0.3$, and $H_{0}=65 \mathrm{~km} \mathrm{~s}^{-1} \mathrm{Mpc}^{-1}$. Under these assumptions the luminosity distance of GRB 050724 is $d_{1}=1400 \mathrm{Mpc}$, and the angular scale corresponds to $4.3 \mathrm{kpc} / \mathrm{arcsec}$.

Section 2 reports the observations carried out for the host galaxy of GRB 050724, and Sect. 3 explains the SED construction method and Sect. 4 reports our results. Section 5 explains our statistical analysis, while Sect. 6 summarises the final conclusions. Our work represents the first statistical attempt to compare the dominant stellar ages of short and long GRB hosts.

\section{Observations and data analysis}

The data were reduced with IRAF $^{1}$ following standard procedures. Table 1 displays the log of our observations. The $U B V R$ band frames were taken with the Andalucía Faint Object and Spectrograph Camera (ALFOSC) at the $2.5 \mathrm{~m}$ Nordic Optical Telescope (2.5NOT). The ALFOSC detector is a $2048 \times 2048$ Thinned Loral CCD providing a field of view (FoV) of 6.5 6.5 and a pixel scale of $0{ }^{\prime} 189 /$ pix. The optical calibration was performed with the $1.5 \mathrm{~m}$ telescope at the Observatorio de Sierra Nevada (OSN) observing a Landolt field at an airmass similar to that of the GRB (Landolt 1992).

The $J_{\mathrm{s}} H K_{\mathrm{s}}$-band observations were carried out with the $3.58 \mathrm{~m}$ Telescopio Nazionale Galileo (3.6TNG) equipped with NICS, a $1024 \times 1024 \mathrm{HgCdTe}$ CCD that provides a FoV of $4 ! 2 \times 4 ! 2$ and a pixel scale of $0.25 /$ pix. The calibration was

${ }^{1}$ IRAF is distributed by the National Optical Astronomy Observatories, which are operated by the Association of Universities for Research in Astronomy, Inc., under cooperative agreement with the National Science Foundation. achieved using eight stars of the 2MASS catalogue that yield a zero point error of $0.03 \mathrm{mag}$ in the $J_{\mathrm{s}} H K_{\mathrm{s}}$ bands. An earlier $J$-band observation was performed on 25.868 UT July 2005 with the $3.5 \mathrm{~m}$ Calar Alto telescope (3.5CAHA) equipped with $\mathrm{OMEGA}_{2000}$. The 3.5CAHA observation was only used for checking the afterglow contamination. Given its large error and the existence of much more accurate $J_{\mathrm{s}}$-band measurements on 4.872 UT and 12.887 UT Aug. 2005, the 3.5CAHA $J$-band magnitude was not used to construct the SED of the host galaxy. We complemented our $U B V R J_{\mathrm{s}} H K_{\mathrm{s}}$-band photometric data points with an $I$-band magnitude $(I=18.68 \pm 0.16)$ obtained from the average of the measurements reported by Berger et al. (2005) and Cobb \& Bailyn (2005).

The observations of the host presented in Table 1 were made at least 11.3 days after the GRB (except the 3.5CAHA data taken on 25.868 UT July 2005). Using $K$-band data, Berger et al. (2005) imposed an upper limit on the afterglow's decay index of $\alpha<-1.9$. Thus assuming a maximum decay index of $\alpha=-1.9$ we estimated an afterglow contribution of at most $\sim 0.02 \%$ to the total light on 4.892 UT Aug. 2005 (our first host galaxy $K_{\mathrm{s}}$-band observing epoch). In addition, the $J$-band magnitude measured with the 3.5CAHA telescope only 1.34 days after the gamma-ray event is consistent with the 3.6TNG $J_{\mathrm{s}}$-band measurements taken on $4.872 \mathrm{UT}$ and 12.887 UT Aug. 2005, supporting (despite its large magnitude error) a low afterglow contamination on our host magnitudes as displayed in Table 1.

\section{Modelling the optical/NIR SED}

The synthetic SED analysis is based on the HyperZ ${ }^{2}$ code (Bolzonella et al. 2000). Eight synthetic spectral types were used: Starburst (Stb), Ellipticals (E), Lenticulars (S0), Spirals $(\mathrm{Sa}, \mathrm{Sb}, \mathrm{Sc}$ and $\mathrm{Sd})$, and Irregular galaxies $(\mathrm{Im})$. Each spectral type has an associated star-formation rate temporal history $S F R(t)$ and a characteristic time scale $S F R \propto \exp (-t / \tau)$ ( $\tau$ ranges from 0 for Stb to $\infty$ for Im galaxies). For the generation of the synthetic templates, three initial mass functions

\footnotetext{
${ }^{2}$ http://webast.ast.obs-mip.fr/hyperz/
} 
(IMFs) were considered: Salpeter (1955, S55), Miller \& Scalo (1979, MS79), and Scalo (1986, S86). Four extinction laws were taken into account: Calzetti et al. (2000, suitable for Stbs), Seaton (1979, for the Milky Way, MW), Fitzpatrick (1986, for the Large Magellanic Cloud, LMC) and Prévot et al. (1984, for the Small Magellanic Cloud, SMC).

For the construction of the synthetic SEDs two metallicity $(Z)$ scenarios were considered: i) a static metallicity model with fixed metallicity values and ii) a dynamic close-box model (Bolzonella et al. 2000). Model ii) considers the galaxy metal enrichment due to ejection of heavy elements by each stellar generation. For Model i) two fixed metallicity values were adopted: $Z=Z_{\odot}$ and $Z=Z_{\odot} / 5$, where $Z_{\odot}=0.02$. The metallicities of i) and ii) diverge with time defining different synthetic templates for evolved stellar populations, especially for large $\tau$ values (i.e. for $\mathrm{E}$ galaxies).

In order to derive the corresponding effective wavelengths and $A B$ magnitudes, we convolved each filter transmission curve, plus the corresponding CCD efficiency curve, with the Vega spectrum (see Table 1). For the construction of the photometric SED, all the magnitudes were corrected for Galactic foreground reddening along the GRB line of sight (Schlegel et al. 1998; $E(B-V)=0.613)$, assuming a typical MW extinction law (Cardelli et al. 1989). Other Galactic $E(B-V)$ values were also tested (see Sect. 4).

\section{Results}

Table 1 shows the optical/NIR magnitudes not corrected for Galactic and host reddening. The magnitudes of the host galaxy are based on the MAG_AUTO magnitude given by SExtractor (Bertin \& Arnouts 1996). The errors in the magnitudes account for both the zero point and the statistical errors.

Given that the spectroscopic redshift is known, we only used the photometric redshift as a sanity check for our SEDs. The accuracy of the photometric redshift determination strongly depends on the number of bands bracketing the rest-frame $\sim 4000 \AA$ break. In our case only the uncertain $B$-band magnitude is at a wavelength that is shorter than $4000 \times(1+z) \AA$, providing a poor coverage of the Balmer break position. Nonetheless the inferred photometric redshift $(z=0.22 \pm 0.03)$ is reasonably consistent with the spectroscopic redshift $(z=0.257)$. Thus hereafter we fix the redshift of the templates at $z=0.257$. Figure 1 shows our photometric points plotted over the SED solution obtained for an MW extinction law, an S86 IMF, and evolving metallicity.

We estimate a mean $M_{B}=-20.7$, corresponding to $L_{B} \sim$ $1.1 L^{\star}$ (Schechter 1976), which is fainter than the $K$-band luminosity $\left(L_{K} \sim 1.6 L^{\star}\right)$ inferred by Berger et al. (2005). We note the uncertainty in our $M_{B}$ value due to the high Galactic reddening. The derived stellar population age ranges from 2.6 to $6.5 \mathrm{Gyr}$ depending on the IMF. The S86 IMF yields systematically better fits than the MS79 and S55 IMFs ( $\chi^{2} /$ d.o.f. 1 for S86 vs. $\chi^{2} /$ d.o.f. $>5$ for MS79 and S55). The S86 IMF is representative of stellar populations with many solar mass stars and few massive stars (Bolzonella et al. 2000). The best fit $\left(\chi^{2} /\right.$ d.o.f. $\left.=0.90\right)$ is obtained with an S86 IMF and an age of $2.6 \mathrm{Gyr}$ (see Fig. 1). This value agrees with (and possibly

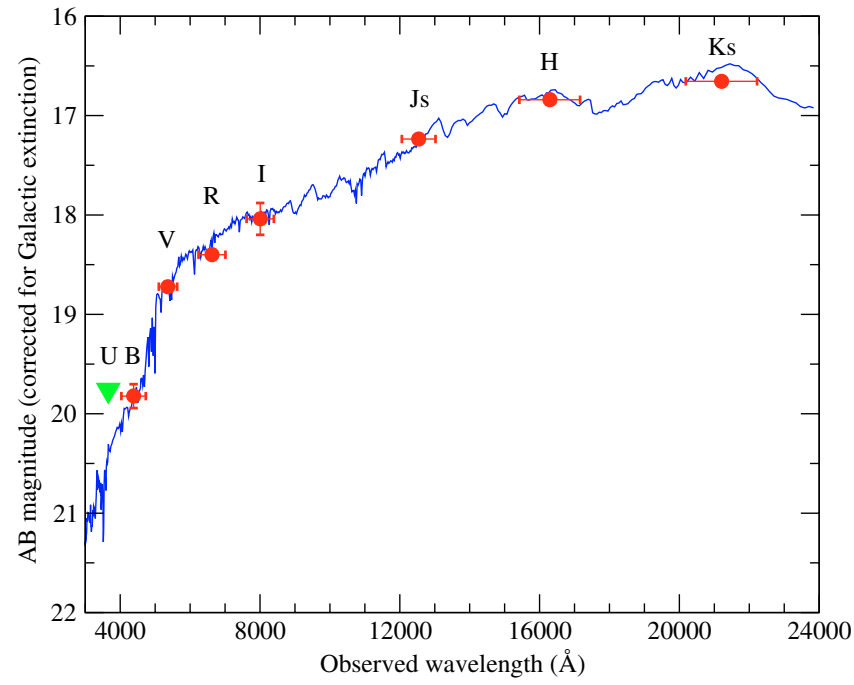

Fig. 1. The optical/NIR SED of the GRB 050724 host. The circles represent the measured magnitudes of the $B V R I J_{\mathrm{s}} H K_{\mathrm{s}}$ bands in the $\mathrm{AB}$ system and corrected for Galactic reddening $(E(B-V)=0.613$; Schlegel et al. 1998). The triangle shows the $U$-band upper limit. The plot shows the satisfactory fit achieved $\left(\chi^{2} /\right.$ d.o.f. $\left.=0.90\right)$ with an S86 IMF, an MW extinction law, and evolving metallicity. The derived stellar population age is $2.6 \mathrm{Gyr}$.

improves) the age lower limit of 1 Gyr estimated by Berger et al. (2005) and Prochaska et al. (2005a), which was based on the absence of strong $\mathrm{H}_{\beta}$ absorption features.

The optical spectra reported for GRB 050724 do not show emission features (Berger et al. 2005; Prochaska et al. 2005a), so a direct measurement of the host galaxy extinction (for instance based on the Balmer decrement; extensively used for long-duration GRB hosts, as well as for GRB 050709; Prochaska et al. 2005a) was not possible. Our optical/NIR SED fitting brings us the opportunity to estimate its value and to hint at whether it could play a crucial role in the faintness of the optical/NIR emission. Moreover if the SEDs of short GRB host galaxies are dominated by old stellar populations (as suggested here), it is plausible that the future spectra of such hosts will frequently not show emission lines either. Thus the SED-fitting technique might become a very valuable tool for estimating extinctions in the hosts of short GRBs.

In the case of the host galaxy of GRB 050724, the inferred mean extinction value is low $\left(A_{\mathrm{v}}=0.2 \pm 0.2\right)$ regardless of the assumed extinction law. This value is similar to the low extinction derived for the host of GRB 050709 (Prochaska et al. 2005a; Hjorth et al. 2005). So in principle the faintness of the optical/NIR afterglow cannot be explained by the global extinction of the host galaxy. But we note that in a few cases dark GRBs have been associated with low extinction hosts (Gorosabel et al. 2003). Given the high foreground Galactic extinction (and its large associated uncertainty), we ranged $E(B-V)$ from 0.613 to 0.265 (as reported by Burstein \& Heiles 1982), thereby extending previous analyses (i.e. Berger et al. 2005) that kept it fixed at the value derived from Schlegel et al. (1998). For $E(B-V)=0.265$ the inferred host galaxy extinction increased to $A_{\mathrm{v}} \sim 0.4$, and the dominant stellar 
population age increased to 5 Gyr. Still the best fit is obtained with an elliptical galaxy, an S86 IMF, and evolving metallicity.

The rest-frame ultraviolet (UV) flux at $2800 \AA$ ( $3520 \AA$ in the observer frame) can provide an estimate of the SFR in a galaxy (Kennicutt 1998). It has been extensively used in SFR calculations of long GRB hosts (Christensen et al. 2004), but for the host galaxy at hand it might result in an overestimate. This is due to the fact that massive galaxies dominated by old stellar populations (age $>1 \mathrm{Gyr}$ ) often show a UV excess (the UV upturn, see Yi \& Yoon 2004, and references therein) originating in the helium-burning stars in the horizontal branch. Hence the proportionality between the UV luminosity and the SFR might not be valid for the host of GRB 050724. When the Kennicutt (1998) relation is applied to our UV flux we obtain an $S F R$ value of $0.5-5 M_{\odot} \mathrm{yr}^{-1}$ (corrected for Galactic and host galaxy extinction), well above the SFR upper limits reported for GRB $050724\left(S F R<0.02 M_{\odot} \mathrm{yr}^{-1}\right.$, Berger et al. 2005; $S F R<0.05 M_{\odot} \mathrm{yr}^{-1}$, Prochaska et al. 2005a, both based on optical spectra). This fact might indicate the presence of an old stellar population. But the uncertain Galactic reddening and inaccurate SED extrapolation to the UV domain (which is highly template dependent) do not permit us to claim a UV upturn for our host.

\section{Discussion: statistical analysis}

Figure 2 shows the distribution of the dominant stellar population ages for two samples of long and short GRB hosts. The sample of long-duration GRB host galaxies contains $N_{1}=$ 13 galaxies, based on Christensen et al. (2004), Gorosabel et al. (2005a), and Chary et al. (2002). We considered a short-duration GRB sample containing only $N_{\mathrm{S}}=4$ host galaxies (GRB 050509B, GRB 050709, GRB 050813, and GRB 050724). To consider this sample we compiled the stellar ages for the host galaxies of GRB 050509B and GRB 050709 and roughly estimated the age of the host of GRB 050813, which has not been reported to date. We used the KolmogorovSmirnov (K-S) test to compare the stellar ages of the long and short GRB host samples. The sample of $N_{\mathrm{s}}=4$ short GRB hosts, although it falls short, is close to the K-S applicability limit $\left(\frac{N_{\mathrm{s}} N_{\mathrm{l}}}{N_{\mathrm{s}}+N_{\mathrm{l}}}=3.06\right.$, Press et al. 1992).

Castro-Tirado et al. (2005) reported a rather young stellar population age $(0.36 \mathrm{Gyr})$ for the host galaxy of GRB 050509B using a photometric BRIJHK-band SED. This age estimate is based on the size of the $4000 \AA$ jump, which requires it to be well bracketed by the SED photometric bands. For the redshift of GRB 050509B $(z=0.225)$, the SED coverage was possibly too reduced to determine the full size of the $4000 \AA$ spectral step. In fact the $4000 \AA$ break (at $\sim 4900 \AA$ for $z=0.225$ ) falls on the outskirts of the $B$-band, the only filter placed on the blue side of the spectral break (see Fig. 2 of Castro-Tirado et al. 2005). Therefore it is plausible that the size of the $4000 \AA$ break, and hence the GRB 050509B host stellar age, were underestimated. This agrees with the lack of star-formation activity and emission lines found in the GRB 050509B spectrum (Bloom et al. 2006) and with the $1 \mathrm{Gyr}$ age upper limit reported by Prochaska et al. (2005a). But for the statistical comparison we decided to use

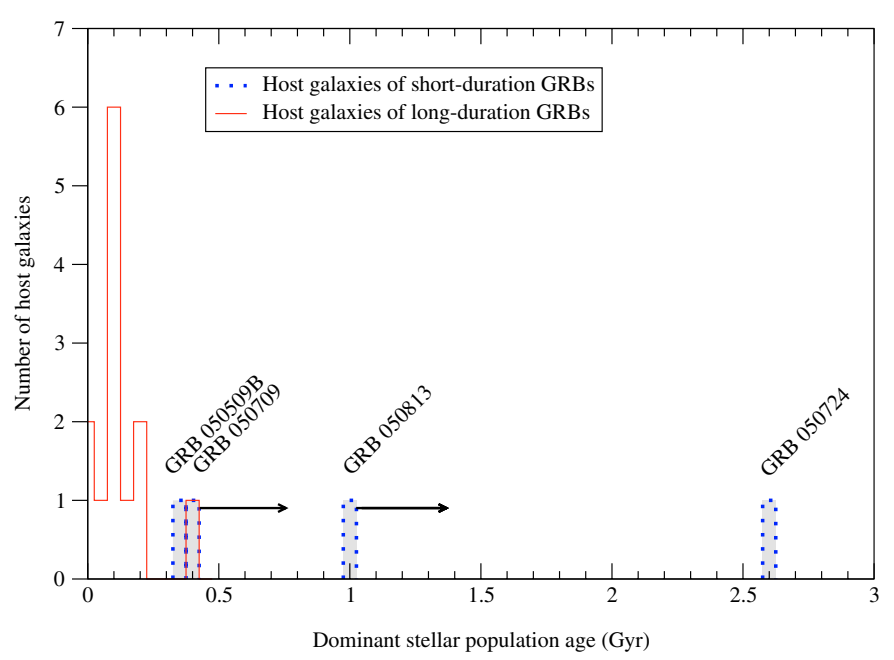

Fig. 2. The distribution of the dominant stellar population ages for short- and long-duration GRB hosts. As shown, host galaxies of short GRBs (filled bins) tend to have older stellar populations than long GRB hosts. According to the WMWW test, the probability that the two samples (short and long GRB host galaxies) share the same parent median age is only $2.5 \times 10^{-3}$. The arrows indicate that the stellar ages assumed for GRB 050509B, GRB 050709, and GRB 050813 are probably underestimated.

the youngest stellar age of $0.36 \mathrm{Gyr}$ reported for the host galaxy of GRB 050509B.

A similar age underestimate cannot be discarded for the host of GRB 050709, for which Hjorth et al. (2005) estimate a rough, dominant stellar age of $0.4 \mathrm{Gyr}$ based on a $B V R I$ band SED. In contrast, Covino et al. (2006) report an older age $(\gtrsim 1 \mathrm{Gyr})$ based on spectral data. For the host galaxy of GRB 050709, as for the GRB 050509B host, we assumed an age lower limit of $0.4 \mathrm{Gyr}$.

The X-ray error box of GRB 050813 is centred in a cluster of galaxies (similar to the host galaxy of GRB 050509B, Pedersen et al. 2005) at $z=0.722$, and contains two red ( $K \sim 19, R-K \gtrsim 4$; Gladders et al. 2005; Prochaska et al. 2005a) elliptical galaxies consistent with the redshift cluster (B and $\mathrm{C}$, following Gorosabel et al. 2005b) and a fainter one with unknown redshift ( $\mathrm{B}^{\star}$, following Prochaska et al. 2005a). In the OSN $I$-band images reported in Gorosabel et al. (2005b) the galaxies B and C show an angular size of $\sim 3-4^{\prime \prime}$, corresponding to a linear diameter of $\sim 25-35 \mathrm{kpc}$ at $z=0.722$. The faintness of the two galaxies in the OSN $I$-band images and the proximity of a bright $\operatorname{star}\left(R \sim 15.2, \sim 10^{\prime \prime}\right.$ to the southeast) prevented a study of their surface brightness profile. Given the linear sizes, colours, and locations of the galaxies B and $\mathrm{C}$ (in the centre of the galaxy cluster) both very likely correspond to massive elliptical galaxies, as suggested by other authors (Gladders et al. 2005; Prochaska et al. 2005a). Cimatti et al. (2002) studied a sample of 78 galaxies with magnitudes $(K \lesssim 19.2)$, colours $(R-K \gtrsim 5)$, and redshifts $(0.7<z<1.5)$ that are similar to the two galaxies located in the GRB 050813 error box, deriving a minimum stellar age of $\sim 3$ Gyr for the 78 galaxies. Thus we assumed a conservative age lower limit of $1 \mathrm{Gyr}$ for the GRB 050813 host stellar population. 
The K-S test gives a probability of $P=3.5 \times 10^{-3}$ that both samples share the same parent age distribution. This statistical comparison strongly suggests that short-duration GRBs occur in host galaxies with older stellar populations than the progenitors of long GRBs. We note that the K-S applicability criterion (described by $\frac{N_{\mathrm{s}} N_{1}}{N_{\mathrm{s}}+N_{1}} \geq 4$ ) is not formally satisfied, since it is currently limited by the low $N_{\mathrm{s}}$ value (but not by $N_{\mathrm{l}}$ ). In order to strictly meet the K-S applicability conditions, it remains a priority to increase the short GRB host sample to $N_{\mathrm{s}} \geq 6$.

To check the impact of the reduced samples on the K-S test, we complemented the analysis with the W Mann-WithneyWilconxon (WMWW) test (Wall \& Jenkins 2003; Siegel \& Castellan 1988). The WMWW test is more appropriate than the K-S test for looking at the differences in the central tendencies of two samples, which seems to be the case for our two host galaxy samples; see in Fig. 2 how short GRB hosts tend to have older ages. This test is non-parametric (unlike other tests, i.e. the T-student test, it does not make any assumptions about the parent distribution), and is suitable for comparing samples with a reduced number of objects. According to the WMWW test, the probability that the two age samples share a parent median is only $P=2.5 \times 10^{-3}$, supporting the $\mathrm{K}-\mathrm{S}$ results.

For long GRB host galaxies there is no correlation between the redshift and the age of the stellar population. Thus the conclusions are unaltered if the sample of short bursts is compared to any subsample of long GRB hosts constructed based on a redshift criterion.

\section{Conclusions}

The optical/NIR SED constructed for the host galaxy of GRB 050724 is reproduced well $\left(\chi^{2} /\right.$ d.o.f. $\left.=0.90\right)$ by a massive $\left(M \sim 5 \times 10^{10} M_{\odot}\right)$ elliptical galaxy with an evolved stellar population (age $\sim 2.6 \mathrm{Gyr}$ ). The best fit is obtained with an S86 IMF, which suggests a stellar population dominated by solarmass stars and with few massive stars. If the metallicity of the host is fixed to a value constant in time, no satisfactory SED fits are achieved $\left(\chi^{2} /\right.$ d.o.f. $>19$ for $\left.Z=Z_{\odot}\right)$. Satisfactory SED fits are obtained only if the host galaxy metallicity increases with time. These findings suggest that GRB 050724 is the consequence of an evolved stellar population consistent with being caused by the merger of two compact objects, a historical prediction of several theoretical models. It is interesting to note that this conclusion joins the ranks of those recently accumulated for other, well-localised, short GRBs. In a broader context our comparative study, based on 4 short- (including GRB 050724) and 13 long-duration GRBs, strongly suggests that the dominant stellar populations of short GRB hosts tend to be older than the ones in host galaxies of long GRBs.

A scenario as simplified as the one presented here is likely to be a naive description of a complex reality. Much as not all long GRB hosts are associated with young, subluminous, starburst galaxies (the spiral hosts of GRB 980425 and GRB 990705 are clear examples, Fynbo et al. 2000; Le Floc'h et al. 2002), not all short GRBs are linked to old, elliptical galaxies (the case of the GRB 050709 host galaxy). The limited sample of short GRB hosts implies that the above conclusions are still governed by small number statistics, and a detailed picture cannot be drawn yet. The identification of new, short-duration, GRB host galaxies will definitively allow for more discriminating statistical comparisons.

Acknowledgements. This research was partially supported by Spain's Ministerio de Educación y Ciencia through programmes ESP2002-04124-C03-01 and AYA2004-01515 (including FEDER funds). J.M.C.C. gratefully acknowledges partial support from the Instrumentcenter for Dansk Astrofysik and the NBI's International $\mathrm{Ph}$.D. School of Excellence. We thank M. Cerviño and J. F. Gómez for helpful discussion of the statistical analysis. We are grateful to C. Coutures and J. Hjorth for their invaluable help. We also wish to thank our anonymous referee for useful comments.

\section{References}

Antonelli, A., Covino, S., Malesani, D., et al. 2005, GCN Circ., 3666 Berger, E., Price, P. A., Cenko, S. B., et al. 2005, Nature, 438, 988 Bertin, E., \& Arnouts, S. 1996, A\&AS, 117, 393

Bloom, J. S., Dupree, A., Chen, H.-W., \& Prochaska, J. X. 2005, GCN Circ., 3672

Bloom, J. S., Prochaska, J. X., Pooley, D., et al. 2006, ApJ, 638, 354

Bolzonella, M., Miralles, J.-M., \& Pelló, R. 2000, A\&A, 363, 476

Burstein, D., \& Heiles, C. 1982, AJ, 87, 1165

Butler, N., Ricker, G., Atteia, J.-L., et al. 2005, GCN Circ., 3570

Calzetti, D., Armus, L., Bohlin, R. C., et al. 2000, ApJ, 533, 682

Cardelli, J.A., Clayton, G. C., \& Mathis, J. S. 1989, ApJ, 345, 245

Castro-Tirado, A. J., de Ugarte Postigo, A., Gorosabel, J., et al. 2005, A\&A, 439, L15

Chary, R., Becklin, E. E., \& Armus, L. 2002, ApJ, 566, 229

Christensen, L., Hjorth, J., \& Gorosabel, J. 2004, A\&A, 425, 913

Cimatti, A., Daddi, E., Mignoli, M., et al. 2002, A\&A, 381, L68

Cobb, B. E., \& Bailyn, C. D. 2005, GCN Circ., 3694

Covino, S., Antonelli, L. A., Romano, P., et al. 2005, GCN Circ., 3665

Covino, S., Malesani, D., Israel, G. L., et al. 2006, A\&A, 447, L5

Fitzpatrick, E. L. 1986, AJ, 92, 1068

Fynbo, J. U., Holland, S., Andersen, M. I., et al. 2000, ApJ, 542, L89

Gal-Yam, A., Nakar, E., Ofek, E., et al. 2005a, ApJ, submitted [arXiv:astro-ph/0509891]

Gal-Yam, A., Cenko, S. B., Berger, E., et al. 2005b, GCN Circ., 3681

Gehrels, N., Barbier, L., Barthelmy, S. D., et al. 2005, Nature, 437, 851

Gladders, M., Berger, E., Morrell, N., \& Roth, M. 2005, GCN Circ., 3798

Gorosabel, J., Andersen, M. I., Hjorth, J., et al. 2002, A\&A, 383, 112

Gorosabel, J., Christensen, L., Hjorth, J., et al. 2003, A\&A, 400, 127

Gorosabel, J., Pérez-Ramírez, D., Sollerman, J., et al. 2005a, A\&A, 444,711

Gorosabel, J., Guziy, S., Sota, A., et al. 2005b, GCN Circ., 3796

Hjorth, J., Sollerman, J., Møller, P., et al. 2003, Nature, 423, 847

Hjorth, J., Watson, D., Fynbo, J. P. U., et al. 2005, Nature, 437, 859

Hurley, K., Berger, E., Castro-Tirado, A. J., et al. 2002, ApJ, 567, 447

Kennicutt, R. C. 1998, ARA\&A, 36, 189

Kouveliotou, C., Meegan, C. A., Fishman, G. J., et al. 1993, ApJ, 413, L101

Landolt, A. U. 1992, AJ, 104, 340

Le Floc'h, E., Duc, P.-A., Mirabel, I. F., et al. 2002, ApJ, 581, L81

Miller, G. E., \& Scalo, J. M. 1979, ApJS, 41, 513

Pedersen, K. P., Elíasdóttir, Á., Hjorth, J., et al. 2005, ApJ, 634, L17

Press, W. H., Teuklosky, S. A., Vetterling, W. T., \& Flannery, B. P. 1992, Numerical recipes in FORTRAN, The art of scientific computing (Cambridge: University Press), 2nd Ed. 
Prévot, M. L., Lequeux, J., Prévot, L., Maurice, E., \& Schlegel, D. J., Finkbeiner, D. P., \& Davis, M. 1998, ApJ, 500, 525 Rocca-Volmerange, B. 1984, A\&A, 132, 389

Seaton, M. J. 1979, MNRAS, 187, 73

Prochaska, J. X., Bloom, J. S., Chen, H.-W., et al. 2005a, ApJ, submitted [arXiv: astro-ph/0510022]

Prochaska, J. X., Chen, H.-W., Bloom, J. S., \& Stephens, A. 2005b, GCN Circ., 3679

Siegel, S., \& Castellan, N. J. 1988, Nonparametric Statistics for the Behavioural Sciences (New York: McGrav-Hill)

Prochaska, J. X., Bloom, J. S., Chen, H.-W., et al. 2005c, GCN Circ., 3700

Retter, A., Barbier, L., Barthelmy, S., et al. 2005, GCN Circ., 3788

Salpeter, E. E. 1955, ApJ, 121, 161

Scalo, J. M. 1986, Fundam. Cosmic Phys., 11, 1

Schechter, P. 1976, ApJ, 203, 297

Stanek, K. Z., Matheson, T., Garnavich, P. M., et al. 2003, ApJ, 591, L17

Wall, J. V., \& Jenkins, C. R. 2003, Practical statistics for Astronomers, handbooks for research astronomers, Vol. 3 (Cambridge: University Press)

Tanvir, N., Chapman, R., Levan, A., \& Priddey, R. 2005, Nature, 438, 991

Yi, S. K., \& Yoon, S.-J. 2004, Ap\&SS, 291, 205 